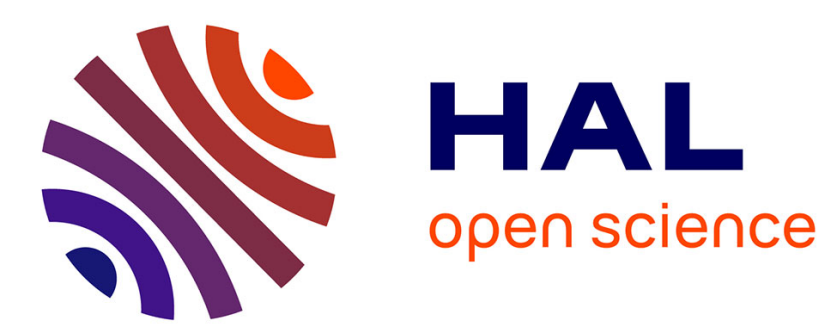

\title{
Augmented Reality for Real-time Navigation Assistance to Wheelchair Users with Obstacles' Management
} Sawssen Ben Abdallah, Faiza Ajmi, Sarah Ben Othman, Sébastien Vermandel, Slim Hammadi

\section{- To cite this version:}

Sawssen Ben Abdallah, Faiza Ajmi, Sarah Ben Othman, Sébastien Vermandel, Slim Hammadi. Augmented Reality for Real-time Navigation Assistance to Wheelchair Users with Obstacles' Management. International Conference on Computational Science, Jun 2019, Faro, Portugal. 10.1007/978-3-03022750-0_47. hal-02439206

\section{HAL Id: hal-02439206 https://hal.science/hal-02439206}

Submitted on 14 Jan 2020

HAL is a multi-disciplinary open access archive for the deposit and dissemination of scientific research documents, whether they are published or not. The documents may come from teaching and research institutions in France or abroad, or from public or private research centers.
L'archive ouverte pluridisciplinaire HAL, est destinée au dépôt et à la diffusion de documents scientifiques de niveau recherche, publiés ou non, émanant des établissements d'enseignement et de recherche français ou étrangers, des laboratoires publics ou privés. 


\title{
Augmented Reality for Real-time Navigation Assistance to Wheelchair Users with Obstacles' Management
}

\author{
Sawssen Ben Abdallah ${ }^{1}$, Faiza Ajmi ${ }^{1}$, Sarah Ben Othman ${ }^{1}$, Sébastien Vermandel ${ }^{2}$, Slim Ham- \\ madi $^{1}$ \\ ${ }^{1}$ CRIStAL Laboratory UMR 9198, Ecole Centrale of Lille, Cité Scientifique, 59651, France \\ ${ }^{2}$ APF France handicap C-RNT, 25 rue Corneille Roubaix 59100, France
}

\begin{abstract}
Despite a rapid technological evolution in the field of technical assistance for people with motor disabilities, their ability to move independently in a wheelchair is still limited. New information and communication technologies (NICT) such as augmented reality (AR) are a real opportunity to integrate people with disabilities into their everyday life and work. AR can afford real-time information about buildings and locations' accessibility through mobile applications that allow the user to have a clear view of the building details. By interacting with augmented environments that appear in the real world using a smart device, users with disabilities have more control of their environment. In this paper, we propose a decision support system using AR for motor disabled people navigation assistance. We describe a real-time wheelchair navigation system equipped with geological mapping that indicates access path to a desired location, the shortest route towards it and identifies obstacles to avoid. The prototyped wheelchair navigation system was developed for use within the University of Lille campus.
\end{abstract}

Keywords: Geological Mapping, Augmented Reality, Wheelchair Users, Short Path, Inclusion, Smart Glasses, Accessibility.

\section{Introduction}

Currently, around $1 \%$ of the general population uses wheelchairs. For 850,000 of these people, motor deficiency is predominant. Despite the existence of several New information and communication technologies (NICT) which can be used to help and assist them, most of the research done concerns sensory disabilities, namely deafness and blindness. However, living conditions improvement for people with motor disabilities could represent an immense hope for them. This improvement includes mobility assistance, which consists of guiding them during their travels. This assistance covers the journey from a source point to a desired destination. It concerns public transport, information on places accessible to people with a wheelchair, tourist information, etc. Each person has some specific criteria for choosing a path while moving from a place to another. Normal people usually choose the shortest path, but disabled people may choose a longer route that does not include an uphill for example. They do not have the ability to make their own choices for their movements. They usually try to memorize routes and accessibility information for every environment they visit which can be problematic in future times if building architecture has been changed, in case of an under-construction road, or the presence of a dynamic or static obstacle that 
could vary from his previous experience. Motor disabled people cannot even easily try a new route because they do not have the information about the surroundings and feel disposed to various kinds of risks such as being blocked in front of a building with no elevator. Many wheelchair users balk to visit a foreign place, as they have no information about the new environment and its accessibility conditions[1]. Some wheelchair users may address these obstacles and others may not. Therefore, obstacle detection becomes an important point while dealing with wheelchair users. Obstacles include narrow aisles, lighting, bad weather, sidewalk width, door handles and/or door pressure, gravel surfaces, etc.[2].

Navigation assistance becomes a crucial point for people with motor deficiency. NICT are a suitable option to improve motor disabled people inclusion in their environment while moving. Among various technologies that exist nowadays, AR technology is suited to make motor disabled people life easier and more comfortable. This technology mixes real and virtual objects using the 3D interaction devices of virtual reality in real space.

\section{Related Work}

In the literature, various studies have been conducted to detach wheelchair users from dependency. They have been working on social inclusion improvements of motor disabled people. In [3], authors have classified motor disabled people into different categories based on their hand stability, and have evaluated various types of interfaces. In [4], a smart wheelchair that can be controlled by gestures has been presented. As well [5] submitted a smart and powered wheelchair based on pattern recognition that could help users become more independent without much physical effort. According to many studies, the number of motor disabled people has been increasing worldwide and there should be solutions able to provide autonomy for wheelchair users. In [6], [7] authors have developed a robotic arm, allowing wheelchair users to individually collect an object from a shelf. [8] has presented an algorithm which returns the optimum path from a source to a destination, and re-orients the user if an obstacle is detected along the path. In fact, navigation path has been studied in robotic movement context [9], [10], [11], where the robot studies all environments and proposes a path to reach the destination while averting any obstacle that can be found in the user environment. Navigation path is based on several approaches such as metaheuristic techniques: genetic algorithm, Dijkstra algorithm, graph theory, etc. These algorithms are designed to provide the optimal path. In [3] authors described an application based on AR and radio frequency identification (RFID) technologies allowing wheelchair users to do shopping and browsing independently with the help of a smartphone or a tablet. Besides, the smart glasses "Glasschair" are used to control the electric wheelchairs with the help head movements ${ }^{1}$.The meaning of the word AR is vast; some of the expert uses a general meaning for it, although others mean some-

1 https://www.rehacare.com/cgi bin/md_rehacare/lib/pub/tt.cgi/GLASSCHAIR_Controlling_wheelchairs_with_smart_glasses.html?oid=46573\&lang=2\&ticket=g_u_e_s_t 
thing very definite. AR, as a technological facilitator, is becoming on top of charts in different fields such as health, education, science, navigation, etc [12].

In the literature, a small number of studies have been dealing with the wheelchair user's requirement using AR. In the previous studies, numerous methods with very different approaches for motor disabled people movement assistance have been proposed. However, none of these systems provides real-time navigation assistance with obstacles management for wheelchairs users.

\section{Solution architecture}

\subsection{Presentation}

With the help of ORA-2 [1] a smart glass that features mobile computing and AR, the ARSAWP (augmented reality system for the assistance of wheelchair people) gives an augmented touch to accessibility information. It is an innovative way to interact between users and accessibility information in the real world; it gives a secure guidance from a place to another. ARSAWP ensures real-time navigation, provides the shortest path to the destination and redirects the user to an emergency route in case of obstacles are encountered.

\subsection{Solution features}

Fig. 1 represents the global architecture of ARSAWP system, which is composed of AR and Android technology combined. Wheelchair user connects via his profile to the ARSAWP system. All of these flows and connections are managed by a connection management system.

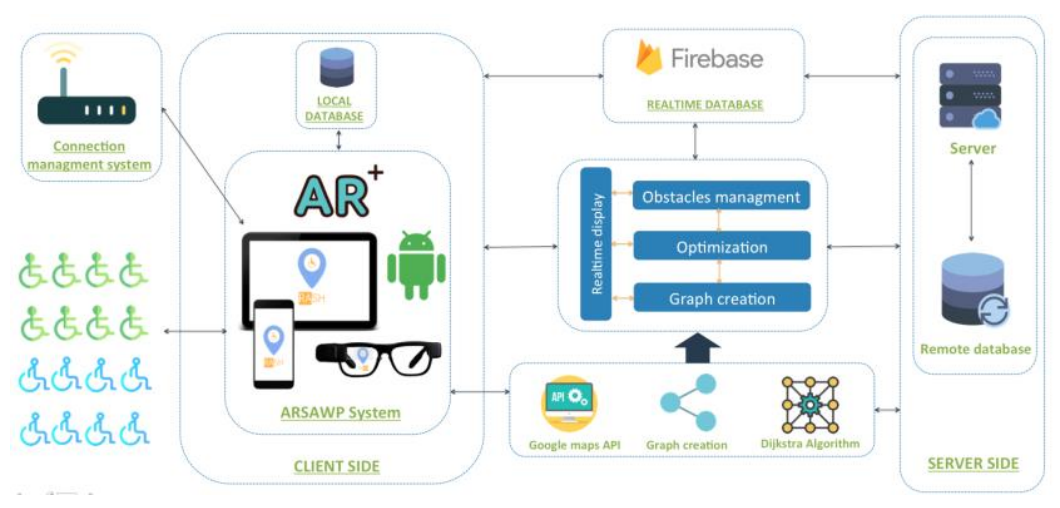

Fig. 1. Global architecture 
Wearing the AR smart glasses, wheelchair user is able to choose a place to go to among several destinations according to his current location. But before that, ARSAWP gives him a glimpse of accessibility information concerning the desired destination whether it is accessible, accessible with assistance or not accessible at all. Once the user has chosen his destination, ARSAWP will proceed to creating the accessibility graph towards the desired location in real-time according to the user's GPS coordinates and the area cartography. We propose an innovative method based on Dijkstra algorithm to calculate the shortest path. In case there's an obstacle blocking the user's way, he can report it. The ARSAWP can then proceed to rerouting to another obstacle-free path. If obstacles are already reported by other users, then obstacles management is handled in terms of notifying wheelchair users of potential obstacles reported that is when a redirection process towards an emergency route is applied by modifying the proposed algorithm based on Dijkstra algorithm. ARSAWP handles a real-time display of obstacles and paths on a map using google maps API. This operation is managed by applying the proposed method based on Dijkstra algorithm alongside accessibility graphs and Google maps API. All these data are stored in a local database and a remote database hosted in a cloud server. Each reported obstacle is recorded in a Firebase, which is a real time database, so that ARSAWP can instantaneously display the obstacles on the map.

\subsection{Methodology implementation}

Fig. 2 shows the proposed algorithm flowchart combined with the real-time behavior of our system. The algorithm ShortPath() returns the optimum path from source to destination. It uses the following arrays:

Visited[i]=1; if the shortest is found from src to i $\mid$ Visited[i]=0; if not

$\mathrm{d}[\mathrm{i}]$ denotes the optimum distance from 'src' to ' $\mathrm{i}$ ' parent[i] denotes the parent of node ' $\mathrm{i}$ ' in the shortest path

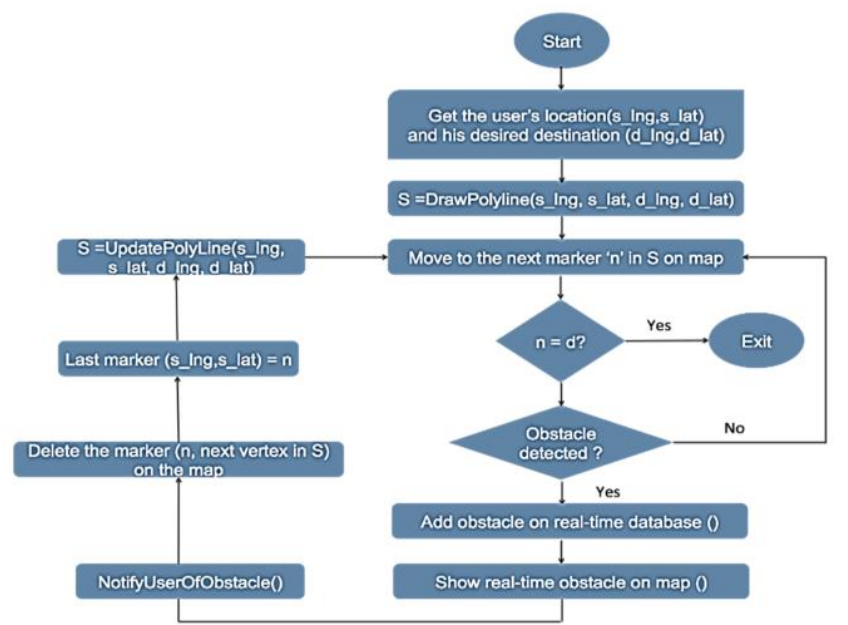

Fig. 2. Algorithm flowchart 


\section{Simulation and results}

Various tests were performed for different scenarios (location not accessible, a route with obstacles and accessible with assistance) in Lille University in the north of France. We considered students using wheelchair to test the developed prototype.

\subsection{User scenario 1: Navigation with obstacles}

In this use case scenario, whenever obstacles have been reported in the path selected by the user, ARSAWP handles obstacles management by notifying him and redirects him towards an alternative route proposed by our proposed algorithms as shown in Fig. 11. At the same time, ARSAWP displays in real-time obstacles and paths on the map that are saved in Firebase, which is a real time database.

\subsection{User scenario 2: Desired destination accessible with assistance}

If the user has to choose a place to go to according to his current location, ARSAWP notifies him that this location is accessible with assistance. For instance, if the entrance door that is not wide enough as shown in Fig. 3, our system suggests finding assistance for him. It can also notify him in real time whether the toilets in the first floor are in service or not. Once arrived at destination, ARSAWP checks if the user has arrived at destination. If this is the case, a gauge of satisfaction is proposed to the user as shown in Fig. 3.

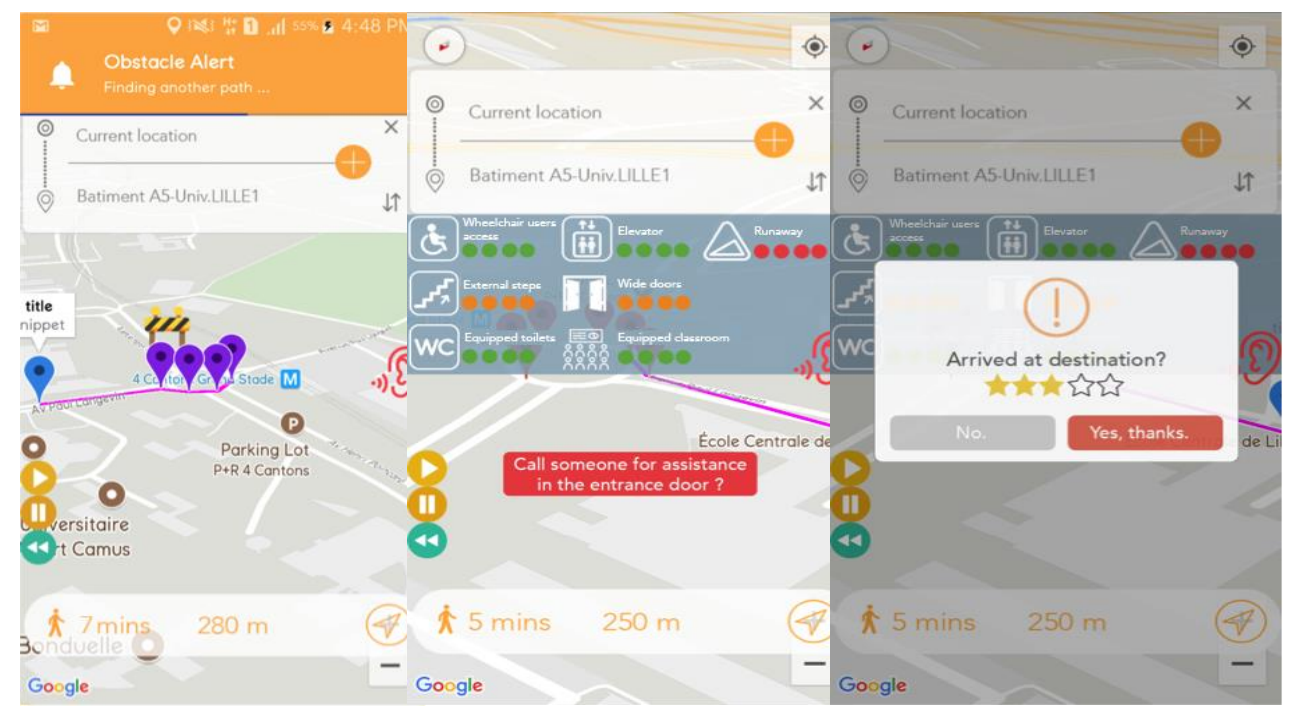

Fig. 3. Navigation system and accessibility information 


\section{Discussion}

ARSAWP system is primarily aimed at providing access information to wheelchair users to avoid creating this wall of not getting the right and useful information. Our solution meets the need of comfort and well being of people with physical and mobility impairments. In fact, after a long and an interesting discussion made with them, they clearly expressed their expectations from this kind of system. Afterwards, we collected all these data and were able to specify the main features of our system which uses AR to increase the accessibility of buildings and locations through a mobile application that allows the user to have a clear view of the building details such as the existence of an elevator, an access ramp in the entrance and if they need assistance in the entrance door. AR makes it possible for users to overcome limitations and difficulties of the environment and allows new ways of understanding it so that wheelchair users will feel at ease. By interacting with augmented environments that appear in the real world using a smart device, users with disabilities have more control of their environment and interact with several information that they could not access before. The proposed algorithm validates the specifications and criteria that a system must provide to wheelchair users in order to ensure a proper route and a smooth navigation from a source to a destination by guiding them through the shortest path in real-time. Besides, using AR and the proposed algorithm we provide the necessary accessibility information about the destination and can notify the users of a blocked path and redirect them to an emergency route for a better guidance. Most importantly, we need to ensure that the next step would be to enhance the developed system to meet the expectations of wheelchair users whom we interviewed.

\section{Conclusion and Perspectives}

ARSAWP is able to help many wheelchair users providing accessibility information and allowing them to navigate freely and surely from a source to a destination in real-time. We aim to make this system available not only for wheelchair users but for all people who present other kind of disabilities, the system will provide guidance according to the user's profile and to the type of disability. The next step will be to make the experience more accurate. In fact, an obstacle verification service has to be included to be certain of the integrity of the obstacle being reported. The best route for people with physical and mobility impairments is not only the shortest distance route from a source to a destination but also the route with minimum of efforts and energy spent that is why distance is negligible compared with time because the more wheelchair users spend time to get to their desired destination the more effort they provide. So taking time into consideration is a must. In addition, we plan to make ARSAWP system more comfortable and easy to use to meet the users' needs by adding vocal guidance as a feature. 


\section{References}

[1] N. Thapar et al., « A pilot study of functional access to public buildings and facilities for persons with impairments », Disability and Rehabilitation, vol. 26, $\mathrm{n}^{\circ}$ 5, p. 280-289, mars 2004.

[2] P. Neis et D. Zielstra, «Generation of a tailored routing network for disabled people based on collaboratively collected geodata », Applied Geography, vol. 47, p. 70-77, févr. 2014.

[3] Z. Rashid, J. Melià-Seguí, R. Pous, et E. Peig, « Using Augmented Reality and Internet of Things to improve accessibility of people with motor disabilities in the context of Smart Cities », Future Generation Computer Systems, vol. 76, p. 248-261, nov. 2017.

[4] Y. Kuno, T. Murashima, N. Shimada, et Y. Shirai, « Interactive gesture interface for intelligent wheelchairs », in 2000 IEEE International Conference on Multimedia and Expo. ICME2000. Proceedings. Latest Advances in the Fast Changing World of Multimedia (Cat. No.00TH8532), 2000, vol. 2, p. 789-792 vol.2.

[5] J. Leaman et H. M. La, «A Comprehensive Review of Smart Wheelchairs: Past, Present, and Future », IEEE Transactions on Human-Machine Systems, vol. 47, $\mathrm{n}^{\mathrm{o}}$ 4, p. 486-499, août 2017.

[6] K. Tsui, D. Kim, A. Behal, D. Kontak, et H. A Yanco, «I Want That": Humanin-the-Loop Control of a Wheelchair-Mounted Robotic Arm », Applied Bionics and Biomechanics, vol. 8, janv. 2011.

[7] S. Kumar, P. Rajasekar, T. Mandharasalam, et S. Vignesh, « Handicapped assisting robot », in 2013 International Conference on Current Trends in Engineering and Technology (ICCTET), 2013, p. 88-91.

[8] D. Nandini et K. R. Seeja, « A novel path planning algorithm for visually impaired people », Journal of King Saud University - Computer and Information Sciences, mars 2017.

[9] J. Minguez et L. Montano, «Sensor-based robot motion generation in unknown, dynamic and troublesome scenarios », Robotics and Autonomous Systems, vol. 52, no 4, p. 290-311, sept. 2005.

[10] R. Yared, X. Défago, J. Iguchi-Cartigny, et M. Wiesmann, «Collision Prevention Platform for a Dynamic Group of Asynchronous Cooperative Mobile Robots », JNW, vol. 2, p. 28-39, 2007.

[11] O. Khatib, «Real-Time Obstacle Avoidance for Manipulators and Mobile Robots », in Autonomous Robot Vehicles, I. J. Cox et G. T. Wilfong, Éd. New York, NY: Springer New York, 1990, p. 396-404.

[12] H. Olmedo, «Virtuality Continuum's State of the Art», Procedia Computer Science, vol. 25, p. 261-270, janv. 2013. 